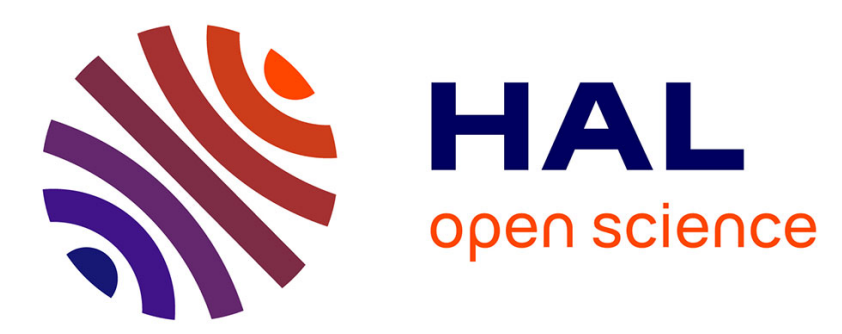

\title{
Une méthode d'étude de phénomènes de compétition entre génotypes. Application au colza (Brassica napus L.)
}

Jean-Marc Azaïs, Janine Onillon, Marianne Lefort-Buson

\section{- To cite this version:}

Jean-Marc Azaïs, Janine Onillon, Marianne Lefort-Buson. Une méthode d'étude de phénomènes de compétition entre génotypes. Application au colza (Brassica napus L.). Agronomie, 1986, 6 (7), pp.601-614. hal-00884915

\section{HAL Id: hal-00884915 https://hal.science/hal-00884915}

Submitted on 1 Jan 1986

HAL is a multi-disciplinary open access archive for the deposit and dissemination of scientific research documents, whether they are published or not. The documents may come from teaching and research institutions in France or abroad, or from public or private research centers.
L'archive ouverte pluridisciplinaire $\mathbf{H A L}$, est destinée au dépôt et à la diffusion de documents scientifiques de niveau recherche, publiés ou non, émanant des établissements d'enseignement et de recherche français ou étrangers, des laboratoires publics ou privés. 


\title{
Une méthode d'étude de phénomènes de compé- tition entre génotypes. Application au colza (Brassica napus L.)
}

\author{
Jean-Marc AZAÏS, Janine ONILLON \& Marianne LEFORT-BUSON $(*$ ) \\ INRA, Laboratoire de Biométrie, route de Saint-Cyr, $F 78000$ Versailles \\ (*) INRA, Station d'Amélioration des Plantes, Centre de Recherches de Rennes, F35650 Le Rheu et \\ CEBA GIS Moulon, F 91190 Gif-sur-Yvette.
}

\begin{abstract}
L'article présente une approche fondamentale et appliquée des phénomènes de compétition entre génotypes et entre parcelles. Dans une $1^{\mathrm{re}}$ partie, les auteurs proposent un modèle complet d'étude de la compétition entre variétés à partir de données de plans en blocs complets dans lesquels les lignes externes et centrales ont été récoltées séparément. Ce modèle est ensuite testé sur des données expérimentales, recueillies sur des colzas de types oléagineux et fourrager, et ramené à un modèle relativement simple. Les résultats confirment l'existence d'effets de compétition intravariétale pour l'espèce ; ils révèlent par ailleurs une liaison entre les effets « producteur » et « compétiteur » de chaque génotype, ainsi qu'une plus grande agressivité des hybrides comparés aux lignées. Dans une $2^{\mathrm{c}}$ partie, les auteurs comparent différents protocoles expérimentaux de récolte et d'analyse des résultats, permettant d'inclure ou non l'effet de la compétition. Le critère de comparaison est basé sur les erreurs quadratiques moyennes des estimateurs de la valeur en plein champ. Dans le cadre restreint des données étudiées il n'est pas apparu très intéressant d'intégrer les phénomènes de compétition intergénotype dans l'analyse. Les auteurs préconisent alors, dans le cas d'essais multilocaux et pluriannuels, un protocole de récolte des seules lignes centrales des parcelles, les lignes externes soumises à la compétition étant éliminées.
\end{abstract}

Mots clés additionnels : Modèle linéaire, résidus autorégressifs, récolte séparée des lignes de bordure, effet compétiteur additif.

This article presents a theoretical and applied study of intergenotype and interplot competition. In the first part a general linear model is proposed for studying intergenotype competition from data of complete block designs in which the inner and external rows of each plot have been separately harvested. The model was tested on fodder rape and rapeseed data, for which the model took a rather simpler form. The results showed the existence of intergenotype competition for rape. They also showed a link between "producer" and "competitor"'effects of the different genotypes. F1 hybrid varieties were more aggressive than selfed lines. In the second part, four different harvest and data analysis procedures are compared for the mean square error of the pure field yield estimators. In the particular case of the rapeseed data, it did not appear useful to introduce a competitor effect into the data analysis. For field trials, over several locations and years, the authors advise harvesting only the inner rows of each plot, the external rows (which have been submitted to competition) being eliminated.

Additional key words : Linear model, autoregressive residuals, separate harvesting of border lines, additive competitor effect.

\section{INTRODUCTION}

Dans les essais en petites parcelles, en amélioration des plantes, l'expérimentateur est souvent confronté aux problèmes de compétition entre génotypes. Les propriétés (par exemple : précision, biais) des estima- teurs des performances de ces génotypes sont affectées par ces phénomènes de compétition. Elles dépendent par ailleurs du protocole de récolte des données et de la méthode utilisée pour leur analyse.

L'article est une contribution à l'étude de ces phénomènes : mise en évidence et incidence sur les esti- 
mations génotypiques. Il se place dans le cadre restreint de résultats expérimentaux de plans en blocs complets dans lesquels les lignes centrales et externes de chaque parcelle ont été récoltées séparément. Les auteurs ont effectué une modélisation du rendement en introduisant différents effets liés à la compétition. La méthode a été utilisée sur le rendement sec (en grain pour le colza oléagineux, en matière sèche pour le colza fourrager) de 3 essais de colza comportant chacun des génotypes homozygotes (lignées) et hétérozygotes (hybrides F1) : 2 essais sur colza oléagineux respectivement d'hiver et de printemps et 1 essai sur colza fourrager.

L'article se compose de 3 parties :

- une présentation mathématique de la terminologie et du modèle utilisé,

- une analyse des résultats des 3 essais avec une comparaison des effets liés à la compétition chez les homozygotes et les hétérozygotes,

- une comparaison théorique de différents protocoles possibles pour un sélectionneur : récolte des seules lignes centrales, récolte de l'ensemble de la parcelle avec et sans introduction d'un effet lié à la compétition, récolte des lignes centrales et externes séparément pour chaque parcelle.

\section{MODÈLE DE L'ÉTUDE}

Nous considérerons des plans en blocs complets dans lesquels les lignes centrales et les lignes externes des parcelles ont été récoltées séparément. Nous appellerons " grandes parcelles » les parcelles originelles du plan en blocs complets, par opposition aux « parcelles élémentaires » constituées :

- soit d'une seule ligne externe d'une grande parcelle, nous parlerons alors de parcelle externe,

- soit de l'ensemble des lignes centrales d'une grande parcelle ; nous parlerons alors de parcelle centrale (fig. 1).

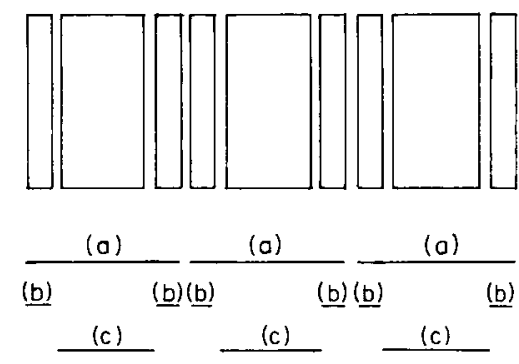

Figure 1

Parcelles élémentaires; elementary plots.

(a) : grandes parcelles; large plots. (b) : parcelles élémentaires externes; external elementary plots. (c): parcelles élémentaires centrales; inner elementary plots.

Nous appellerons producteur le génotype semé sur la parcelle élémentaire.

\section{A. Hypothèses}

Nous supposerons les parcelles suffisamment allongées pour ne considérer les voisinages que dans une direction. Une parcelle élémentaire n'a donc que 2 voisins. Dans une au moins de ces parcelles élémentaires (fig. 2), on retrouve le même génotype que le producteur. Nous appellerons compétiteur l'autre génotype voisin (pour les parcelles centrales il est identique au producteur). Nous supposerons enfin que le comportement d'une ligne n'est influencé que par les 2 lignes immédiatement voisines.

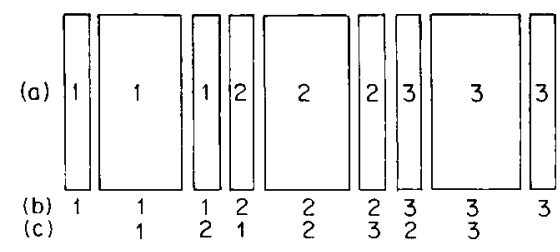

Figure 2

Prodicteur et comperiteur ; producer and compethor. (a) : numéro du génotype: genotype number. (b) : producteur; producer. (c) : compétiteur; competitor.

Enfin nous noterons :

$\mathrm{p}$ : le nombre de lignes d'une grande parcelle,

$\mathrm{r}$ : le nombre de répétitions,

$\mathrm{n}$ : le nombre de génotypes.

\section{B. Le modèle}

Nous allons modéliser le rendement des parcelles. Notre démarche peut s'appliquer sans problème à d'autres variables. Le modèle classique pour l'analyse des résultats des grandes parcelles comprend un effet du bloc et un effet du producteur. Soit $Y_{i j}$ la moyenne des rendements des lignes (ou le rendement à l'hectare) du génotype i dans le bloc j. En notation indicielle il s'écrit :

$$
\begin{aligned}
& \mathrm{Y}_{\mathrm{ij}}=\mu+\mathrm{BL}_{\mathrm{j}}+\mathrm{PR}_{\mathrm{i}}+\varepsilon_{\mathrm{ij}} \\
& \mathrm{i}: \text { producteur } \\
& \mathrm{j}: \text { bloc }
\end{aligned}
$$

les erreurs $\varepsilon_{\mathrm{ij}}$ étant indépendantes et de même variance.

Quand il n'y aura pas de risque d'ambiguité, nous noterons nos modèles de manière factorielle, sans préciser les indices. Le modèle (A), par exemple, sera noté :

$$
\mathrm{Y}=\mu+\mathrm{BL}+\mathrm{PR}+\varepsilon .
$$

Nous allons présenter nos modèles comme des extensions du modèle (A) bien connu des améliorateurs. Pour une présentation plus générale, plus précise et plus mathématique, on se reportera à AZAIS et al. (1984).

Les données de parcelles élémentaires sont de 2 natures suivant qu'elles correspondent à une parcelle centrale ou à une parcelle externe. Elles ne pourront être comparées que si le rendement est ramené soit à l'ha soit à la ligne. 


\section{Modélisation de l'espérance (effets fixes)}

Pour prendre en compte les phénomènes liés à la compétition, nous pouvons ajouter au modèle (A), modèle qui peut s'appliquer également aux données de parcelles élémentaires, les différents effets suivants :

- un effet additif du compétiteur, $C$, qui mesure l'effet moyen en tant que compétiteur d'un génotype vis-à-vis de tous les autres.

- un effet « depression par la compétition », DC, qui prend 2 valeurs selon qu'il s'agit d'une parcelle centrale ou d'une parcelle de bordure. Il est la traduction du phénomène bien connu suivant : le rendement d'un génotype sur une ligne externe, où il est en condition de compétition, est souvent inférieur au rendement sur une parcelle centrale où il n'est en voisinage qu'avec lui-même. Bien sûr, le signe de cet effet n'est pas précisé. L'effet DC peut également modéliser le phénomène inverse,

- une interaction DC-BL, nous avons en effet constaté que l'importance de l'effet de dépression variait d'un bloc à l'autre. Cela peut s'interpréter en disant que l'intensité de la compétition est liée à l'abondance des ressources dans le sol, abondance qui peut varier d'un bloc à l'autre,

- une interaction entre le producteur et la dépression par la compétition, PR-DC. La mise en évidence d'une valeur élevée de cette interaction soulignerait l'existence d'une différence spécifique à chaque génotype entre le rendement en voisinage de soi-même (censé représenter le rendement en plein champ qui est le paramètre d'intérêt) et le rendement en conditions de compétition. Il serait alors impératif de ne pas utiliser les lignes externes pour l'estimation de la valeur en plein champ,

- une interaction entre le producteur et le compétiteur. En général le nombre de répétitions est trop faible pour pouvoir introduire dans le modèle une interaction entre le produteur et le compétiteur : cela demanderait $\mathrm{n}^{2}$ degrés de liberté au total. Dans la partie expérimentale qui suit, les génotypes de colza considérés sont issus d'un plan de croisement diallèle complet. Dans ce cas particulier, il est possible d'introduire des interactions entre les généalogies du producteur et du compétiteur ; ainsi les effets producteur et compétiteur peuvent être décomposés en :

- un effet lignée pure ou hybride qui permet de modéliser l'hétérosis. Cet effet est noté LP ou LC selon qu'il est associé au producteur ou au compétiteur,

- un effet héritable des parents noté respectivement $\mathrm{PP}$ et $\mathrm{PC}$,

- un effet résiduel noté respectivement PR et $C$.

C'est la décomposition classique du diallèle qui s'écrit par exemple pour le producteur :

$$
\begin{aligned}
& \mathrm{PR}_{\mathrm{i}}=\mathrm{LP}_{\mathrm{m}(\mathrm{i})}+\mathrm{PP}_{\mathrm{k}(\mathrm{i})}+\mathrm{PP}_{l(\mathrm{i})}+\mathrm{PR}_{\mathrm{i}} \\
& \mathrm{k}(\mathrm{i}) \text { : père du génotype } \\
& \text { 1(i) : mère du génotype } \\
& \text { m(i) : } 0 \text { pour une lignée pure }
\end{aligned}
$$

Cette décomposition permet en particulier d'étudier l'influence de l'hétérosis sur les effets producteur et compétiteur ainsi que l'héritabilité de ces effets, mais elle permet surtout l'étude des interactions symbolisées par, LP-PC ; PP-LC ; LP-LC ; PP-PC.

\section{Structure de variance (effets aléatoires)}

Dans le modèle (A) sur les grandes parcelles, les erreurs sont indépendantes et de même variance; la transposition de ce modèle aux données de parcelles élémentaires implique des variances différentes pour les parcelles centrales et externes. En effet, les données recueillies sur les parcelles centrales sont des moyennes de ( $\mathrm{p}-2)$ lignes. Il faut donc pondérer les variances par :

- (p-2) pour une parcelle centrale,

-1 pour une parcelle externe.

Dans tout ce qui suit nous supposerons que cette pondération a été faite une fois pour toutes et nous ne la rappellerons plus.

Par rapport à cette structure qui correspond au cas simple (ou à l'hypothèse nulle si l'on veut se placer dans la philosophie des tests), 2 types de différences peuvent se rencontrer :

- inégalités de variances (malgré la pondération). Les parcelles externes représentent un volume expérimental plus faible que les parcelles centrales. Les erreurs techniques (pertes au battage, imprécision de pesées) peuvent être plus importantes. On doit alors avoir soin de vérifier que les variances des données de parcelles externes et centrales sont bien dans le rapport prévu par la pondération,

- autocorrélation entre erreurs; pour modéliser une situation où les erreurs associées à 2 parcelles différentes sont d'autant plus liées que les 2 parcelles sont plus proches, on utilise un processus autorégressif d'ordre 1 (qui est défini par cor $\left(\varepsilon_{n}, \varepsilon_{n+h}\right)=\alpha^{h}$, avec $\alpha<1$ ). Nous prenons ici en, compte la partie aléatoire (indépendante des génotypes) de la compétition. De manière imagée, on pourrait parler de compétition entre parcelles et non plus de compétition entre génotypes ; par exemple, une parcelle peut germer plus tôt et occuper de manière décisive le sol, les parcelles voisines sont alors déprimées.

\section{Estimation et test dans le cadre du modèle présenté}

La réunion de tous les effets précédemment cités conduit à l'élaboration du modèle le plus général que nous noterons (B). La difficulté d'étude d'un tel modèle réside dans l'estimation et les tests à la fois sur les paramètres de l'espérance et sur ceux de la structure de variance des termes d'erreurs.

Nous avons utilisé la démarche simplifiée suivante: traiter le modèle (B) sous l'hypothèse nulle pour la structure de variance des erreurs la plus simple (résidus indépendants et de même variance), puis tester les différents effets par les techniques de régression descendante (DRAPER \& SMITH, 1981). Dans ce cas le modèle (B) est un modèle linéaire non orthogonal classique; on peut utiliser pour le calcul un logiciel de régression comme la directive "regress " de Genstat (ASTIER el al., 1982) ou MOdLl (KOBILINSKY, 1982). Enfin pour le modèle retenu, on testera, d'une part, la présence d'autocorrélations par un test de DurbinWatson (cf. annexe 1) et, d'autre part, l'égalité des 
variances en comparant les moyennes des carrés des résidus (par résidus nous entendons les estimations des termes d'erreurs) associés, d'une part, aux parcelles centrales et, d'autre part, aux parcelles externes. Si ces 2 quantités sont très éloignées de l'égalité, on doit rejeter l'hypothèse d'égalité. Il est par contre difficile de donner une valeur seuil exacte.

N.B. Même si les erreurs $\epsilon$ ont même variance, il n'en est pas de même pour les résidus e. Si X est la matrice du modèle, le calcul matriciel montre que : $\operatorname{Var}(\mathrm{e})=\left(\mathrm{I}_{\mathrm{N}}-\mathrm{X}\left(\mathrm{X}^{\prime} \mathrm{X}\right)^{-1} \mathrm{X}^{\prime}\right) \sigma^{2}(\mathrm{~N}$ est le nombre total d'observations). La méthode ci-dessus reste approximative si on n'utilise pas les résidus réduits, calculés en particulier par le logiciel MODLI. Si $\sigma_{i j}^{2}$ est le terme ij de la matrice Var (e), le ième résidu réduit vaut $\mathrm{e}_{\mathrm{i}} / \sigma_{\mathrm{ii}}$

\section{APPLICATION DES MÉTHODES À DES DONNÉES RECUEILLIES SUR DES PARCELLES EXPÉRIMENTALES DE COLZA}

\section{A. Présentation des données}

Des études antérieures simples avaient montré l'existence d'effets de compétition entre génotypes chez le colza oléagineux d'hiver (BUSON, 1979). Cet auteur a alors utilisé des dispositifs expérimentaux mis en place pour l'étude de la vigueur hybride sur le colza oléagineux d'hiver (LEFORT-BUSON \& DATTÉE, 1985) et de printemps (LEFORT-BUSON, 1982) ainsi que sur le colza fourrager (LEFORT-BUSON, 1983), pour confirmer l'existence de la competition entre génotypes chez différents types de colza.

L'étude de la vigueur hybride a été effectuée en utilisant des plans de croisement diallèle entre lignées d'origines diverses. Les dispositifs expérimentaux étaient des plans en blocs quasi complets (dans certains blocs, un génotype manque, il est alors remplacé par le témoin) à 4 répétitions, avec des parcelles de 5 lignes de $3 \mathrm{~m}$, espacées de $0,3 \mathrm{~m}$. La grande parcelle, définie au chapitre précédent, est ainsi constituée de 3 parcelles élémentaires de dimensions différentes : la ligne externe gauche, les 3 lignes centrales et la ligne externe droite.

Les 3 parcelles élémentaires sont récoltées et pesées indépendamment, un échantillon est prélevé pour déterminer leur teneur en matière sèche. Le rendement est alors calculé et ramené en $\mathrm{q} / \mathrm{ha}$. Les données expérimentales de rendement sec correspondant aux 3 essais précédemment définis (colza oléagineux d'hiver (1), de printemps (2) et colza fourrager (3)), sont présentées en annexe.

\section{B. Résultats des analyses}

\section{Modélisation de l'espérance}

Après analyse statistique, les 3 essais ont conduit au même modèle :

$$
\begin{aligned}
\mathrm{Y}= & \mu+\mathrm{BL}+\mathrm{DC}+\mathrm{BL}-\mathrm{DC}+\mathrm{LP}+ \\
& \mathrm{PP}+\mathrm{PR}+\mathrm{LC}+\mathrm{PC}+\varepsilon \cdot(\mathrm{C})
\end{aligned}
$$

Pour poser un modèle général qui s'applique à des génotypes quelconques (non issus d'un plan de croisement diallèle) nous pouvons regrouper les différents effets issus de la décomposition des effets producteur et compétiteur :

$$
\mathrm{Y}=\mu+\mathrm{BL}+\mathrm{DC}+\mathrm{BL}-\mathrm{DC}+\mathrm{PR}+\mathrm{C}+\varepsilon .
$$

\section{Structure de variance des erreurs}

Les tests de Durbin-Watson ont toujours été non significatifs; ils ne permettent pas de remettre en cause l'indépendance des erreurs. Pour les 2 premiers essais, nous avons aussi conclu à l'égalité des variances ; en revanche dans l'essai $n^{\circ} 3$, la variance des parcelles externes a été estimée 43 fois plus importante que celles des parcelles centrales. Cela souligne le peu de fiabilité qui doit être accordée aux données récoltées sur une seule ligne pour du colza fourrager. Dans la suite nous interpréterons les résultats correspondant à cet essai avec précaution.

$N$. B. Dans la suite de cet article, nous considérerons que, dans le modèle (D), les résidus sont indépendants et de même variance.

\section{Commentaires}

Dans le modèle (D), et avec les contraintes qui sont précisées en annexe, une quantité intéressante à estimer pour le génotype $i$ est la quantité $P R_{i}+C_{i}$ qui représente (à une constante près due aux termes $\mathrm{DC}$, BL-DC, constante qui ne modifie pas les comparaisons entre génotypes) le rendement du génotype i en voisinage de lui-même; elle correspond a priori aux performances en plein champ et peut être retenue pour représenter la valeur génotypique. Nous la noterons $t_{i}$.

L'effet producteur $\mathbf{P R}_{\mathrm{i}}$ s'interprète par contre comme la valeur moyenne du génotype quand il est bordé par l'ensemble des autres génotypes de l'essai.

L'effet compétiteur $C_{i}$ représente le biais dû à la compétition : c'est la différence entre la valeur $\mathbf{P R}_{\mathrm{i}}$, mesurée par une expérience où intervient la compétition, et la valeur que l'on cherche $P_{\mathrm{i}}+\mathrm{C}_{\mathrm{i}}$. Enfin $\left(-C_{i}\right)$ peut également être interprété comme l'agressivité du génotype $i$.

- La significativité d'effets extérieurs au modèle (A), DC, BL-DC, C, montre clairement, s'il en était besoin, la présence d'effets de compétition chez le colza.

- Tous les effets complexes (interactions entre généalogies, LP-LC, LP-PC, PP-LC, PP-PC, ou interaction producteur-dépression par la compétition, $\mathrm{PR}$ DC) se sont avérés non significatifs, ce qui conduit à un modèle relativement simple.

- L'interaction entre le bloc et la dépression, BL$D C$, est significative, ce qui montre une grande sensibilité des phénomènes de compétition vis-à-vis de l'environnement.

- L'effet du compétiteur est entièrement expliqué par la généalogie.

- Les effets LP et LC sont toujours significatifs : l'hétérosis s'exprime fortement sur l'effet producteur et l'effet compétiteur ; en moyenne les hybrides ont un rendement en plein champ $\left(\mathrm{PR}_{\mathrm{i}}+\mathrm{C}_{\mathrm{i}}\right)$ plus élevé 

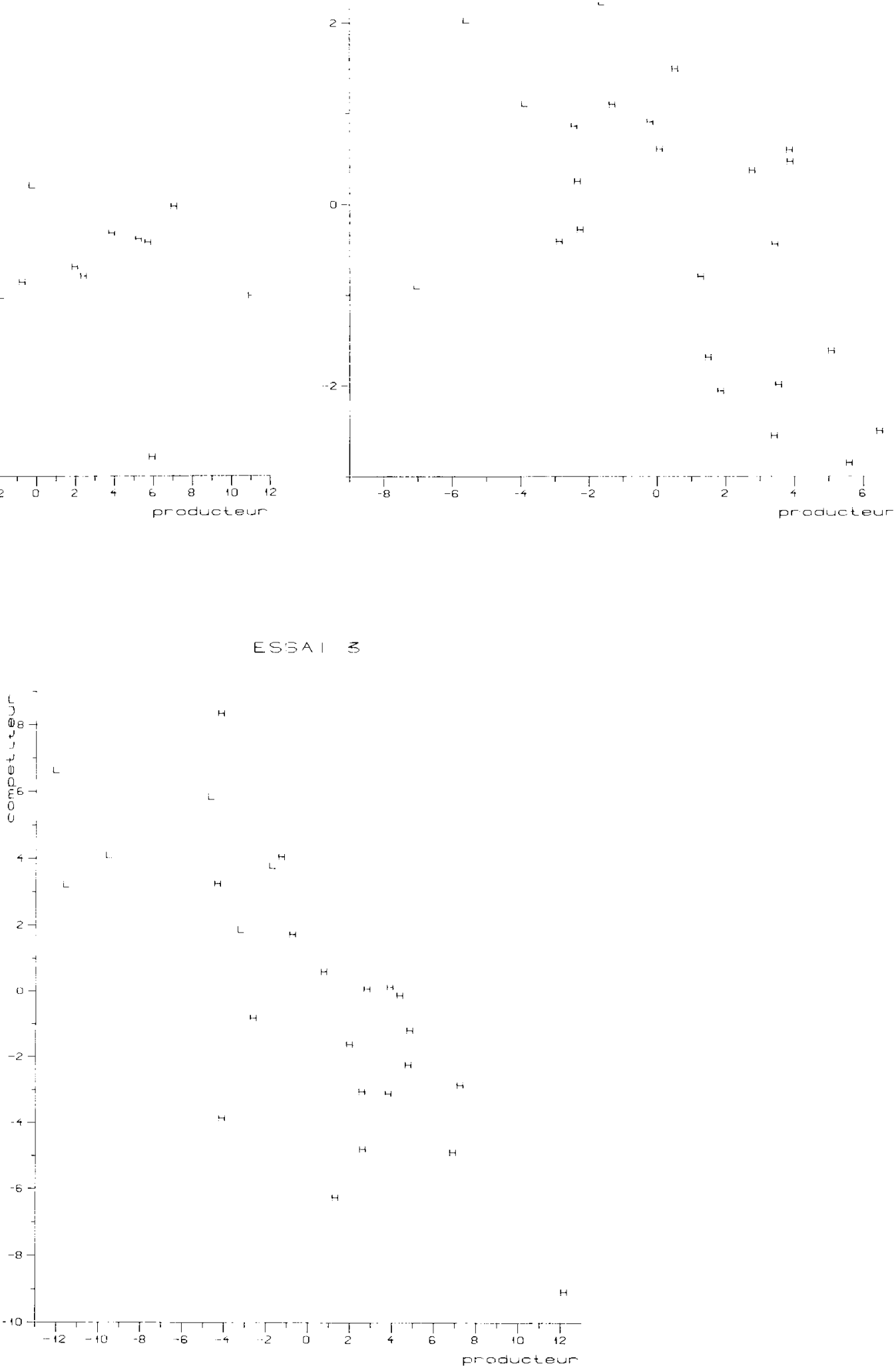

Figure 3

Essais I, 2, 3. Effet producteur contre effet compétiteur. Les valeurs sont exprimées en q/ha. L : lignée pure; $H:$ hybride. Producer effect against competitor effect. Values are in quintals $[100 \mathrm{~kg} /$ per hectare. $L:$ selfed line; $H:$ hybrid variety. 
que celui des lignées pures; ils sont également plus agressifs : LC est négatif pour un hybride.

\section{Valeurs numériques}

Les valeurs estimées des différents effets (producteur, compétiteur, valeur en plein champ) sont présentées dans l'annexe 3. Seuls sont donnés les graphiques des effets producteur contre compétiteur (fig. 3). Ils montrent une liaison négative, pour l'ensemble des génotypes, entre les 2 effets ; la liaison persiste intrahybride ou intra-lignées sauf peut-être pour les hybrides des données (1). Indépendamment de la structure homozygote ou hétérozygote, plus un génotype est producteur (PR positif) plus il est agressif ( $\mathrm{C}$ négatif). Nous n'avons pas représenté les graphiques valeur en plein champ contre effet compétiteur ; la liaison négative est beaucoup moins nette et ne persiste pas intrahybride ou intra-lignées.

\section{COMPARAISONS DE DIVERS PROTOCOLES DE RÉCOLTE ET D'ANALYSE À PARTIR DU MODÈLE (D)}

\section{A. Introduction}

Nous proposons 4 protocoles différents pour la récolte et l'analyse d'un plan en blocs complets. Nous les comparons dans le cadre particulier des essais précédemment cités. Le protocole qui doit être considéré comme le meilleur est celui qui permet d'estimer au mieux les valeurs en plein champ des différents génotypes. Malheureusement plusieurs critères peuvent être utilisés ; nous reviendrons sur ce problème au chapitre (IV.E). Sur les 4 protocoles présentés, les 2 premiers sont très souvent utilisés en amélioration des plantes :

(1) Récolte des seules parcelles centrales; les données sont analysées suivant le modèle associé au plan en blocs complets.

(2) Récolte des grandes parcelles en totalité, sans tenir compte de la compétition ; l'analyse est la même que précédemment.

(3) Récolte identique au protocole (2) ; mais on introduit un effet du compétiteur dans l'analyse.

(4) Récolte séparée de toutes les parcelles élémentaires : centrales et externes; les données sont analysées suivant le modèle (D).

Hormis le protocole (4) ces protocoles sont de coûts relativement égaux.

\section{B. Critère de comparaison des différents protocoles}

Le protocole (2) donne des estimateurs biaisés : on sélectionne partiellement sur l'aptitude à la compétition. Pour prendre en compte les biais notre critère sera basé sur des erreurs quadratiques moyennes et non pas sur des variances. Si $\hat{\mathrm{t}}$ est un estimateur :

$$
\operatorname{EQM}(\hat{\mathrm{t}})=\operatorname{biais}^{2}(\hat{\mathrm{t}})+\operatorname{Var}(\hat{\mathrm{t}})
$$

Par ailleurs, nous recherchons un critère qui se situe sur l'espace des contrastes entre traitements pour éli- miner le problème constant de confusion de la moyenne générale avec tous les autres effets. Notre critère $\gamma$ sera proportionnel à la moyenne, sur tous les couples de génotypes, des erreurs quadratiques moyennes (EQM) des estimateurs des contrastes élémentaires, $t_{i}-t_{j}$ :

$$
\gamma=1 / 2 \mathrm{n} \sum_{\substack{\mathrm{i}=1, \mathrm{n} \\ j=1, n}} \operatorname{EQM}\left(\hat{\mathrm{t}}_{\mathrm{i}}-\hat{\mathrm{t}}_{\mathrm{j}}\right)
$$

Le meilleur protocole est celui qui correspond au plus faible $\gamma$, en tenant compte toutefois que, dans certaines situations, le biais des estimateurs du protocole (2) peut être rédhibitoire.

Dans le cas où $\sum_{\mathrm{i}=1, \mathbf{n}} \mathrm{t}_{\mathrm{i}}=0=\sum_{\mathrm{i}=1, \mathrm{n}} \hat{\mathrm{t}}_{\mathrm{i}}$, le calcul montre :

$$
\gamma=\sum_{i=1, n} \operatorname{VAR}\left(\hat{\mathrm{t}}_{\mathrm{i}}\right)+\operatorname{biais}^{2}\left(\hat{\mathrm{t}}_{\mathrm{i}}\right)
$$

\section{Calcul du critère pour les différents protocoles}

Pour exposer les méthodes de calcul du critère $\gamma$, il est nécessaire de détailler le modèle (D) qui inclut les modèles des 4 protocoles et de préciser les hypothèses qui ont été faites. Le détail est donné dans l'annexe 1. nous tenons toutefois à exposer une condition qui nous paraît importante :

\section{Condition de circularité des blocs}

Pour rendre le modèle (D) proche de l'orthogonalité et pour simplifier son traitement numérique, il serait agréable de pouvoir considérer que les 2 grandes parcelles extrêmes d'un bloc sont voisines. La condition serait vérifiée par exemple, si les blocs étaient des couronnes et les parcelles disposées comme sur la figure 4 :

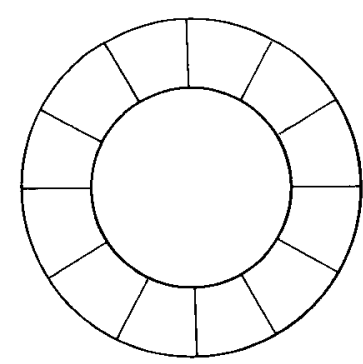

Figure 4

Bloc en couronne; crown block.

Cette figure bien sûr n'est pas réaliste, mais la condition peut être vérifiée en bordant chaque bloc par le génotype semé à l'autre extrémité du bloc (fig. 5).

Cette condition n'est pas fondamentale mais, dans toute étude de la compétition, il nous semble préférable de border les blocs comme présenté dans la figure 5 plutôt qu'avec un témoin fixe. Dans ce qui suit, nous supposerons que la condition de circularité des blocs est vérifiée. 


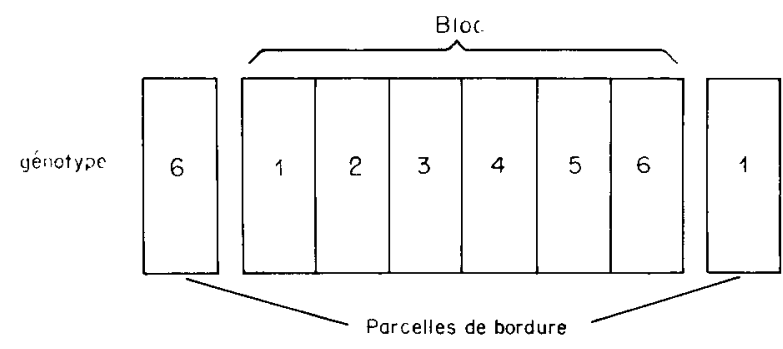

Figure 5

Comment rendre vraie en pratique la condition de circularité des blocs; how to make the circularity condition for the blocks true in practice.

\section{Résultats numériques relatifs aux essais sur le colza oléagineux}

Les expressions du critère sont relativement complexes et dépendent, pour le protocole (2), des paramètres du modèle. Nous n'avons fait de comparaisons que dans les cas particuliers des essais (1) et (2) : plans en blocs complets à 4 répétitions, pour 16 et 25 génotypes, avec des grandes parcelles de 5 lignes. Les résultats sont indiqués dans le tableau 1.

Nous nous intéresserons à la moyenne du critère sur toutes les randomisations; ceci nous paraît cohérent pour des plans non systématiques. Hormis les valeurs correspondant au protocole (2), toutes les valeurs du tableau 5 sont proportionnelles, le facteur de proportionnalité étant connu, à la variance résiduelle : $\sigma^{2}$. On peut donc considérer toutes les différences entre les valeurs correspondant aux protocoles (1), (3) et (4) comme significatives.

\section{E. Interprétation}

Chez le colza, l'introduction d'un effet compétiteur, dans l'analyse de résultats d'essais variétaux, n'apporte pas grand chose : le protocole (3) semble donc sans intérêt. Le protocole (4) ne semble pas non plus apporter une amélioration suffisante pour compenser son coût plus élevé. Pour le protocole (2) une mise en garde s'impose. Nous avons montré au chapitre III que l'effet du compétiteur était significatif. Les estimations qu'il fournit sont donc biaisées : dans un programme de sélection où l'on va multiplier le même essai en différents lieux et années, la moyenne des estimations ne convergera pas vers la valeur en plein champ du génotype mais vers la valeur en condition de compétition $\left(\mathrm{PR}_{\mathrm{i}}\right)$. Cela peut être grave. A notre sens, il ne faut l'utiliser que dans une expérience qui sera peu ou non répétée : dans ce cas, le biais est noyé dans la variance qui reste importante. Dans ce cas précis il faudra même l'employer de préférence car il donne les meilleurs résultats. Dans les autres cas on utilisera le protocole (1).

\section{CONCLUSION}

L'analyse des résultats a souligné des effets de compétition élevés entre les lignes externes de 2 parcelles adjacentes chez le colza, oléagineux et fourrager. La modélisation de ces effets s'est avérée relativement simple.

La compétition s'exprime par une agressivité plus ou moins forte des génotypes lorsqu'ils sont placés les uns à côté des autres ; l'agressivité semble être liée linéairement à l'effet producteur. Les hybrides qui ont un effet producteur supérieur à celui des lignées sont donc plus agressifs que ces dernières ; ce résultat avait déjà été pressenti par BUSON (1979), qui attribuait aux hybrides une meilleure aptitude à la compétition.

Pour le sélectionneur dont le but est d'approcher au mieux la valeur en plein champ d'un génotype à partir d'estimations microparcellaires, se pose alors la question de prendre ou non en compte ces effets de compétition. Sur le colza, les résultats obtenus dans cette étude permettent de discuter de cette dernière éventualité ; ils demandent cependant à être confirmés avant de conclure définitivement.

Si l'on veut réaliser une seule expérience isolée sur un même ensemble de variétés, il ne semble pas utile de tenir compte de la compétition. Pour des expériences répétées en différents lieux et années, les comparaisons intervariétales effectuées en petites parcelles semblent devoir être faites en éliminant les effets de compétition par la récolte des seules lignes centrales, et ce d'autant plus que les différences entre génotypes sont plus élevées. La récolte des lignes centrales, d'une part, et des lignes externes, d'autre part, ne conduit pas dans cet exemple à un réel gain de précision, compte tenu du surcoût de l'expérimentation.

Reçu le 30 janvier 1985. Accepté le 10 mars 1986.

\section{TABLEAU 1}

Valeur du critère pour les différents protocoles.

Value of the criterion for the different procedures.

\begin{tabular}{lcccc}
\hline \hline & $\begin{array}{c}\text { (1) Parcelles centrales } \\
\text { seulement }\end{array}$ & $\begin{array}{c}\text { (2) Grandes parcelles } \\
\text { analyse } \\
\text { blocs compl. }\end{array}$ & $\begin{array}{c}\text { (3) Grandes parcelles } \\
\text { analyse } \\
\text { eff. compét. }\end{array}$ & (4) Parcelles élémentaires \\
\hline Colza oléag. d'hiver (1) & 92,4 & $76,1\left(^{*}\right)$ & $164,2\left(^{*}\right)$ & $71 \quad(*)$ \\
\hline Colza oléag. printemps (2) & 31,1 & $25,6\left(^{*}\right)$ & $49,3\left(^{*}\right)$ & $23,8(*)$ \\
\hline
\end{tabular}




\section{ANNEXE 1}

\section{Calcul du critère pour les différents protocoles}

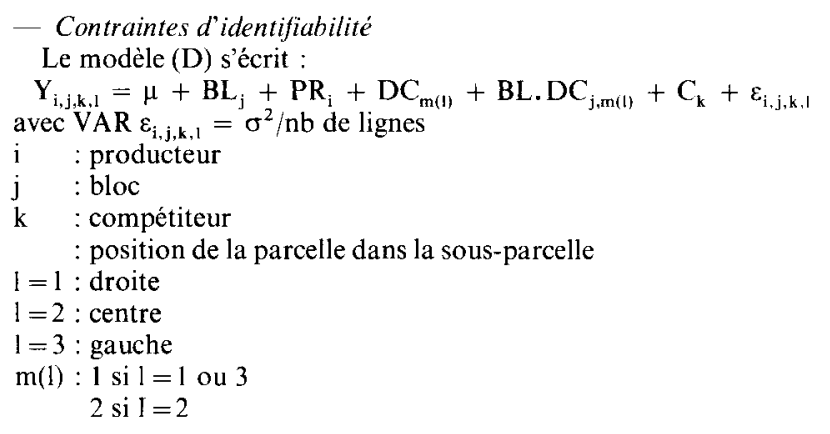

Pour que les paramètres du modèle soient déterminés de manière unique, il faut leur imposer des contraintes. Pour des commodités de calcul nous avons choisi les suivantes :

$$
\begin{aligned}
& \sum_{j=1, \mathrm{n}} \mathrm{BL}_{\mathrm{j}}=0 ;(\mathrm{p}-2) \mathrm{DC}_{2}+2 \mathrm{DC}_{1}=0 ; \sum_{\mathrm{j}=1, \mathrm{n}} \mathrm{BL} \cdot \mathrm{DC}_{\mathrm{jm}}=0 ; \\
& (p-2) B L \cdot D C_{j 2}+2 \text { BL. } D C_{1}=0 ; \sum_{i=1, n} P_{i}=0 ; \sum_{k=1 . n} C_{k}=0 \text {. }
\end{aligned}
$$

Ces contraintes combinées avec la condition de circularité des blocs rendent le modèle presque orthogonal : seuls les effets producteur et compétiteur ne le sont pas.

- Les sous-modèles du modèle (D) qui correspondent aux divers protocoles

- Le modèle (D) s'applique aux données du protocole (4).

- Dans les protocoles (2) et (3), les grandes parcelles sont récoltées en entier. Si on regroupe les 3 données d'une grande parcelle dans le modèle (D) on obtient :

$$
\begin{gathered}
\mathrm{Y}_{\mathrm{i}, \mathrm{j}, \mathbf{k}, \mathbf{k}^{\prime}}=\mu+\mathrm{BL}_{\mathrm{j}}+\mathrm{PR}_{\mathrm{i}}+1 / \mathrm{p}\left(\mathrm{C}_{\mathrm{k}}+\mathrm{C}_{\mathrm{k}^{\prime}}+(\mathrm{p}-2) \mathrm{C}_{\mathrm{i}}\right)+\varepsilon_{\mathrm{i}, \mathrm{j}}^{(2)}(\mathrm{E}) \\
\operatorname{Var} \varepsilon^{(2)}=\sigma^{2} / \mathrm{p}
\end{gathered}
$$

\section{i : producteur}

j : bloc

$\mathrm{k}, \mathrm{k}^{\prime}$ : couple des 2 compétiteurs d'une grande parcelle

Posons :

il vient :

$$
\begin{aligned}
\tilde{\mathrm{P}}_{\mathrm{i}} & =\mathrm{PR}_{\mathrm{i}}+(\mathrm{p}-2) / \mathrm{p \textrm {C } _ { \mathrm { i } }} \\
\tilde{\mathrm{C}}_{\mathrm{k}} & =\mathrm{C}_{\mathrm{k}} / \mathrm{p}
\end{aligned}
$$

$$
Y_{i j k k^{\prime}}=\mu+B L_{j}+\tilde{\mathbf{P}}_{i}+\tilde{C}_{k}+\tilde{C}_{k^{\prime}}+\varepsilon_{i j}^{(2)}
$$

C"est le modèle qui sera utilisé pour le protocole (3)

- Pour le protocole (2) sur le même modèle, on utilisera comme estimateur de la valeur en plein champ $t_{i}\left(=\widetilde{P}_{i}+2 \widetilde{C}_{i}\right)$, l'estimateur classique mais biaisé : $\hat{t}_{i}=Y_{i}-Y_{\text {. (le }}$. désigne la moyenne sur l'indice qu'il remplace).

- Pour le protocole (1) on ne récolte que les parcelles centrales En restreignant le modèle (D) à $1=2$, on obtient :

$$
\begin{aligned}
Y_{i j} & =\mu+B L_{j}+D C_{2}+B L \cdot D C_{2 j}+P_{i}+C_{i}+\varepsilon_{i j}^{(3)} \\
& i \text { : producteur } \\
& j: \text { bloc }
\end{aligned}
$$

qui peut se réécrire :

$$
Y_{i j}=\tilde{\mu}+B L_{j}+T_{i}+\varepsilon_{i j}^{(3)}
$$

$t_{i}$ est estimé par : $Y_{i .}-Y_{\text {.. }}$

\section{- Calcul du critère}

Mis à part le cas du protocole (2), le calcul se limite à celui de la trace de la matrice de variance des $\hat{t}_{\mathrm{i}}$.

- Il n'est simple que pour le protocole (1)

$$
\gamma=\sigma^{2}(\mathrm{n}-1) /(\mathrm{p}-2) \mathrm{r}
$$

\section{$\mathrm{n}$ : nombre de génotypes; $\mathbf{r}$ : nombre de répétitions.}

- Pour les protocoles (3) et (4), on ne peut obtenir qu'une expression matricielle dont on peut calculer informatiquement la valeur (nous avons utilisé le langage APL). L'expression est la suivante :

$$
\gamma=\operatorname{TR}\left(\mathbf{C}^{\prime}\left(\mathbf{X}^{\prime} \mathbf{X}+\mathrm{H}^{\prime} \mathrm{H}\right)^{-1} \mathbf{X}^{\prime} \mathbf{X}\left(\mathbf{X}^{\prime} \mathbf{X}+\mathrm{H}^{\prime} \mathrm{H}\right)^{-1} \mathrm{C}\right)\left\{\begin{array}{l}
\sigma^{2} \\
\sigma^{2} / \mathbf{P}
\end{array}\right.
$$

$$
\begin{aligned}
& X: \text { matrice d'incidence }: Y=X \theta+\varepsilon \\
& H: \text { matrice des contraintes }: H \theta=0 \\
& C: \text { matrice définissant les } t_{i} C^{\prime} \theta=\left(t_{1}, \ldots, t_{n}\right)^{\prime} .
\end{aligned}
$$

Dans ces 2 cas, le résultat est aléatoire : il dépend de la randomisation au travers des voisinages entre les génotypes dans les différents blocs. Pour le protocole (3) la dépendance est forte et sa forme a résisté à notre analvse : il ne suffit pas par exemple d'imposer que 2 génotypes ne se retrouvent jamais voisins plus d'une fois, ou qu'un couple de compétiteurs n'apparaisse jamais plus d'une fois comme les compétiteurs d'une même grande parcelle, pour que le critère s'abaisse de manière notable. Pour le protocole (4), la variabilité due à la randomisation est négligeable.

- Pour le protocole (2), on montre ci-dessous qu'en moyenne sur toutes les randomisations possibles :

$$
\gamma=(\mathrm{n}-1) \sigma^{2} / \mathrm{pr}+2 \mathrm{n} / \mathrm{r}\left\lceil\frac{3(\mathrm{n}-1)+2(\mathrm{r}-1) \mathrm{n}}{(\mathrm{n}-1)^{2}}\right\rceil \mathrm{Q} / \mathrm{p}^{2}
$$

$\operatorname{avec} Q=\sum_{k=1, n} C_{k}^{2}$

Le modèle exact pour le protocole (2) est le modèle (E)

$$
\mathrm{Y}_{i, \mathrm{j}} ; \mathrm{k}_{1}, \mathrm{k}_{2}=\mu+\mathrm{BL}_{\mathrm{j}}+\tilde{\mathrm{P}}_{\mathrm{i}}+\tilde{\mathrm{C}}_{\mathrm{k}_{1}}+\tilde{\mathrm{C}}_{\mathrm{k}_{2}}+\varepsilon_{\mathrm{ij}}^{(2)}
$$

nous considérons les estimateurs $\hat{\mathrm{t}}_{\mathrm{i}}=\mathrm{Y}_{\mathrm{i}}-\mathrm{Y}_{\text {.. }}$.

\section{- Notations}

$b_{i j}=\widetilde{C}_{k_{1}}+\tilde{C}_{k_{2}}-2 \widetilde{C}_{i}$ que l'on peut appeler le « biais dans le bloc $\mathrm{j}$ "

$$
\text { bi }=\text { biais de } Y_{i .}-Y_{.} .
$$

Il est facile de voir que $\mathrm{bi}=\mathrm{bi}$.

Comme $\Sigma \hat{\mathrm{t}}_{\mathrm{i}}=0=\Sigma \mathrm{t}_{\mathrm{i}}$

$$
\gamma=\sum_{\mathrm{i}} \operatorname{Var}\left(\mathrm{Y}_{\mathrm{i} .}-\mathrm{Y}_{. .}\right)+\Sigma \mathrm{b}_{\mathrm{i}}^{2}=\frac{(\mathrm{n}-1) \sigma^{2}}{\mathrm{pr}}+\Sigma \mathrm{b}_{\mathrm{i}}^{2} .
$$

Il reste à calculer $\Sigma b_{i}^{2}=A$.

A est une variable aléatoire puisqu'elle dépend de la randomisation du plan en blocs complets, nous allons calculer $E(A)$ sous cette randomisation. Nous allons d'abord calculer l'espérance d'un $b_{i}^{2}: E\left(b_{i}^{2}\right)$

$$
E\left(b_{i}^{2}\right)=\frac{1}{r^{2}}\left[\sum_{j} E\left(b_{i j}^{2}\right)+\sum_{j \neq j^{\prime}} E\left(b_{i j} b_{i j^{\prime}}\right)\right] .
$$

Comme $b_{i j}$ et $b_{i j}$ sont 2 variables aléatoires déterminées par 2 randomisations intrabloc indépendantes :

$$
E\left(b_{i j} b_{i j}\right)=E\left(b_{i j}\right) E\left(b_{i j}\right)
$$

Un calcul montre que $E\left(b_{i j}\right)=-\frac{2 n}{(n-1)} \tilde{C}_{i j}$.

On en déduit : $E\left(b_{i}^{2}\right)=\frac{1}{r^{2}} \sum_{j} E\left(b_{i j}^{2}\right)+\frac{4(r-1) n^{2}}{r(n-1)^{2}} \tilde{C}_{i}^{2}$.

Calculons $E\left(b_{i j}^{2}\right)$

$$
b_{i j}^{2}=\left(\tilde{\mathrm{C}}_{\mathrm{k}_{1}}^{2}+\check{\mathrm{C}}_{\mathrm{k}_{2}}^{2}+4 \tilde{\mathrm{C}}_{\mathrm{i}}^{2}-2 \tilde{\mathrm{C}}_{\mathrm{k}_{1}} \tilde{\mathrm{C}}_{\mathrm{k}_{2}}-4 \tilde{\mathrm{C}}_{\mathrm{k}_{1}} \tilde{\mathrm{C}}_{\mathrm{i}}-4 \tilde{\mathrm{C}}_{\mathrm{k}_{2}} \tilde{\mathrm{C}}_{\mathrm{i}}\right)
$$

Conditionnons par un des compétiteurs $\mathrm{k}_{1}$, en remarquant que :

$$
\mathrm{E}\left(\tilde{\mathrm{C}}_{\mathrm{k}_{2}} / \mathrm{k}_{1}\right)=-\frac{\tilde{\mathrm{C}}_{\mathrm{k}_{1}}-\tilde{\mathrm{C}}_{\mathrm{i}}}{\mathrm{n}-2} ; \mathrm{E}\left(\tilde{\mathrm{C}}_{\mathrm{k}}^{2} / \mathrm{k}_{1}\right)=\frac{\overline{\mathrm{Q}}-\tilde{\mathrm{C}}_{\mathrm{k}_{1}}^{2}-\tilde{\mathrm{C}}_{\mathrm{i}}^{2}}{\mathrm{n}-2}
$$

$\operatorname{avec} \widetilde{\mathrm{Q}}=\sum_{\mathrm{k}} \widetilde{\mathrm{C}}_{\mathrm{k}}^{2}$

$\mathrm{E}\left(\mathrm{b}_{\mathrm{ij}}^{2} / \mathrm{k}_{1}\right)=\left[\frac{\mathrm{Q}}{\mathrm{n}-2}+\frac{4 \mathrm{n}-5}{\mathrm{n}-2} \tilde{\mathrm{C}}_{\mathrm{i}}^{2}+\frac{10-4 \mathrm{n}}{\mathrm{n}-2} \widetilde{\mathrm{C}}_{\mathrm{i}} \mathrm{C}_{\mathrm{k}_{1}}+\frac{\mathrm{n}-5}{\mathrm{n}-2} \tilde{\mathrm{C}}_{\mathrm{k}_{1}}^{2}\right]$.

On déconditionne par rapport à $\mathrm{k}_{\downarrow}$

$$
\begin{aligned}
& E\left(\tilde{\mathrm{C}}_{\mathrm{k}_{1}}\right)=-\frac{\tilde{\mathrm{C}}_{\mathrm{i}}}{\mathrm{n}-1} ; \mathrm{E}\left(\tilde{\mathrm{C}}_{\mathrm{k}_{\mathrm{L}}}^{2}\right)=\frac{\tilde{\mathrm{Q}}-\tilde{\mathrm{C}}_{\mathrm{i}}}{\mathrm{n}-1} \\
& \mathrm{E}\left(\mathrm{b}_{\mathrm{ij}}^{2}\right)=\left[\frac{2(\mathrm{n}-3)}{(\mathrm{n}-2)(\mathrm{n}-1)} \tilde{\mathrm{Q}}+\frac{2(\mathrm{n}-3) \mathrm{n}}{(\mathrm{n}-2)(\mathrm{n}-1)} \widetilde{\mathrm{C}}_{\mathrm{i}}^{2}\right]
\end{aligned}
$$

d'où

$E\left(b_{i}^{2}\right)=\frac{1}{r}\left[\frac{2(n-3) \tilde{Q}}{(n-2)(n-1)}+\frac{4(r-1) n(n-2)+2(2 n-3) n-1}{(n-2)(n-1)^{2}} n \tilde{C}_{i}^{2}\right]$ 
en sommant

$E(\gamma)=\frac{(n-1) \sigma^{2}}{p r}+\frac{2 n}{r}\left[\frac{2 r n+n-3}{(n-1)^{2}}\right] \frac{Q}{r^{2}}$

Pour estimer Q sur les données de colza, nous avons "débiaisé 》 l'estimateur :

$\hat{\mathrm{Q}}=\sum_{\mathrm{k}=1, \mathrm{n}} \hat{\mathrm{C}}_{\mathrm{k}}^{2}$; on sait que :

$$
\mathrm{E}(\hat{\mathrm{Q}})=\left(\sum_{\mathrm{k}=1, \mathrm{n}} \mathrm{C}_{\mathrm{k}}^{2}\right)+A \sigma^{2}
$$

A est la trace de la matrice de variance des $\hat{C}_{k}$ qui peut être calculée à laide de l'informatique. On utilise alors l'estimateur :

$\hat{\mathrm{Q}}=\sum_{\mathbf{k}=1, \mathrm{n}} \hat{\mathrm{C}}_{\mathrm{k}}^{2}-\hat{\mathrm{A}} \sigma^{2}$ quand cette quantité est positive, sinon $\hat{\mathrm{Q}}=0$.

\section{ANNEXE 2}

\section{Test de Durbin-Watson}

Le test de Durbin-Watson est un test sous l'hypothèse de normalité de l'hypothèse nulle d'indépendance des erreurs contre l'hypothèse générale d'erreurs autorégressives d'ordre 1. Son utilisation pose dans notre cas 2 problèmes :

(1) Sauf dans le cas où les blocs sont bout à bout, les parcelles ne sont pas naturellement ordonnées et les erreurs ne sont autorégressives qu'à l'intérieur des blocs et indépendantes d'un bloc à l'autre. Nous proposons d'ordonner les blocs les uns à la suite des autres de manière quelconque. On vérifie que l'hypothèse nulle correspond bien à celle du test de Durbin-Watson, tandis que l'hypothèse générale est légèrement différente. Le test reste de niveau exact mais il perd peut-être de ses propriétés d'optimalité.

(2) On ne dispose de tables pour le test de Durbin-Watson que dans le cas où il y a peu de régresseurs : 5 degrés de liberté au plus dans le modèle. Ce n'est bien sûr pas le cas. Il existe heureusement plusieurs méthodes d'approximation (DURBIN \& WATSON, 1950, $1951,1971)$ pour pouvoir calculer les valeurs seuils. Nous avons choisi la bêta-approximation car son principe nous a paru le plus simple. Nous la détaillons ci-dessous. Nous supposons les parcelles ordonnées et nous noterons $\mathrm{e}_{\mathrm{m}}$ le résidu, c'est-à-dire l'estimation de $\varepsilon_{\mathrm{in}}$, de la même parcelle. La statistique de test est définie par

$$
d=\frac{\sum_{m=2}^{N}\left(e_{m}-e_{m-1}\right)^{2}}{\sum_{m=1}^{N}\left(e_{m}\right)^{2}}
$$

$\mathrm{N}$ est le nombre de parcelles ( $\mathrm{N}=3 \mathrm{nr}$ dans notre cas). La statistique d est comprise entre 0 et 4 avec une valeur « centrale » de 2 . Si d est petit le test conclut à des erreurs autocorrélées positivement, si $\mathrm{d}$ est grand négativement, la méthode consiste à approximer la distribution de $\mathrm{d}$ sous l'hypothèse nulle par la loi Bêta de même espérance et variance. On déterminera les paramètres de cette loi de la façon suivante :

Soit X la matrice d'incidence du modèle linéaire

$$
\mathrm{Y}=\mathbf{X} \theta+\varepsilon
$$

Soit $(\mathrm{k}-1)$ le nombre de degrés de liberté

Alors $E(d)=\frac{1}{N-k}\left[2(N-1)-\operatorname{tr}\left\{\left(X^{\prime} A X\right)\left(X^{\prime} X\right)^{-1}\right\}\right]$

A est la matrice $\mathrm{N} \times \mathrm{N}$

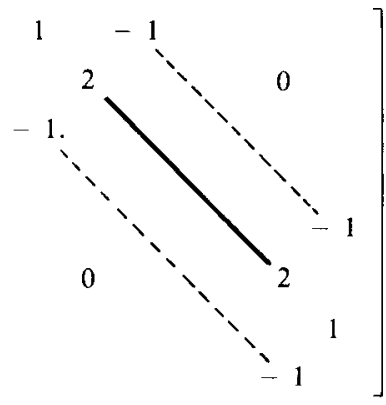

$$
\operatorname{Var}(\mathrm{d})=\frac{2}{(\mathrm{n}-\mathrm{k})(\mathrm{n}-\mathrm{k}+2)}\left\{\mathrm{Q}-(\mathrm{E}(\mathrm{d}))^{2}(\mathrm{n}-\mathrm{k})\right\}
$$

avec

$Q=2(3 n-4)-2 \operatorname{tr}\left\{X^{\prime} A^{2} X\left(X^{\prime} X\right)^{-1}\right\}+\operatorname{tr}\left[\left\{X^{\prime} A X\left(X^{\prime} X\right)^{-1}\right\}^{2}\right]$.

On détermine $p$ et $q$ de la loi Bêta par :

$$
\begin{aligned}
\mathrm{p}+\mathrm{q} & =\frac{\mathrm{E}(\mathrm{d})\{4-\mathrm{E}(\mathrm{d})\}}{\operatorname{Vard}} \\
\mathrm{p} & =\frac{1}{4}(\mathrm{p}+\mathrm{q}) \mathrm{Ed} .
\end{aligned}
$$

Sous $H_{0}: F=\frac{p(4-d)}{q d} \sim F_{2 p, 2 q}$ loi de Fischer de paramètres 2 p, 2 q.

En considérant respectivement $\mathrm{F}$ ou $1 / \mathrm{F}$ on teste l'hypothèse nulle contre les hypothèses unilatérales : erreurs autocorrélées positivement (respectivement négativement). 
ANNEXE 3

Données des essais

Donमe日s: $\cdot 1=$

Eolza oLea日
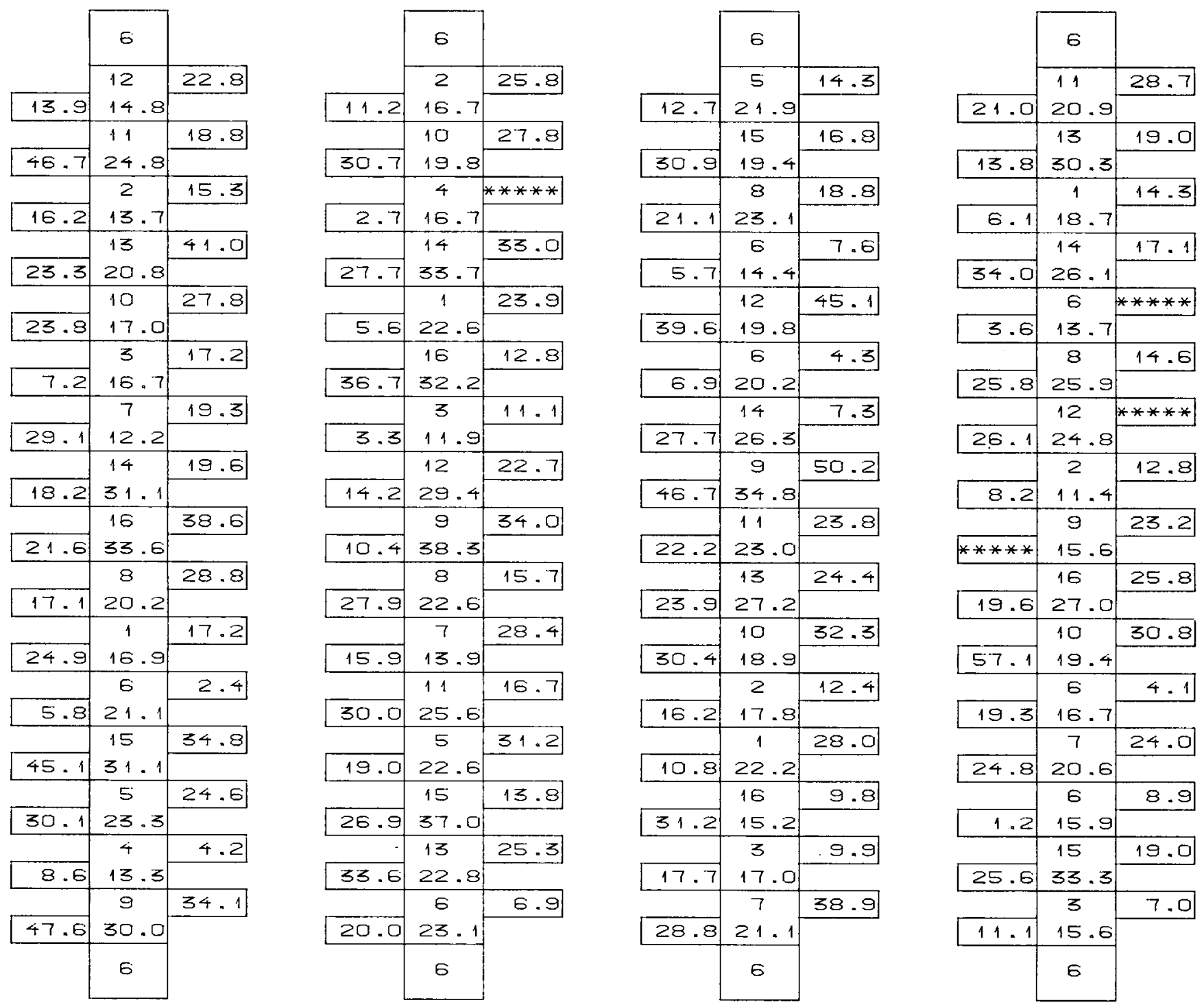

Dans le rectangle central en haut est inscrit le numéro du génotype ; ailleurs sont inscrits les rendements des parcelles élémentaires ramenés en q/ha. Les données considérées comme suspectes ont été soulignées.

In the upper rectangle is found the genotype number; elsewhere are found yield of elementary plots in quintals per hectare. Outliers have been underlined. 
Danne日s $\cdot 2 \cdot z=$

COLzo OLeOGLn日ux

de prLntemps
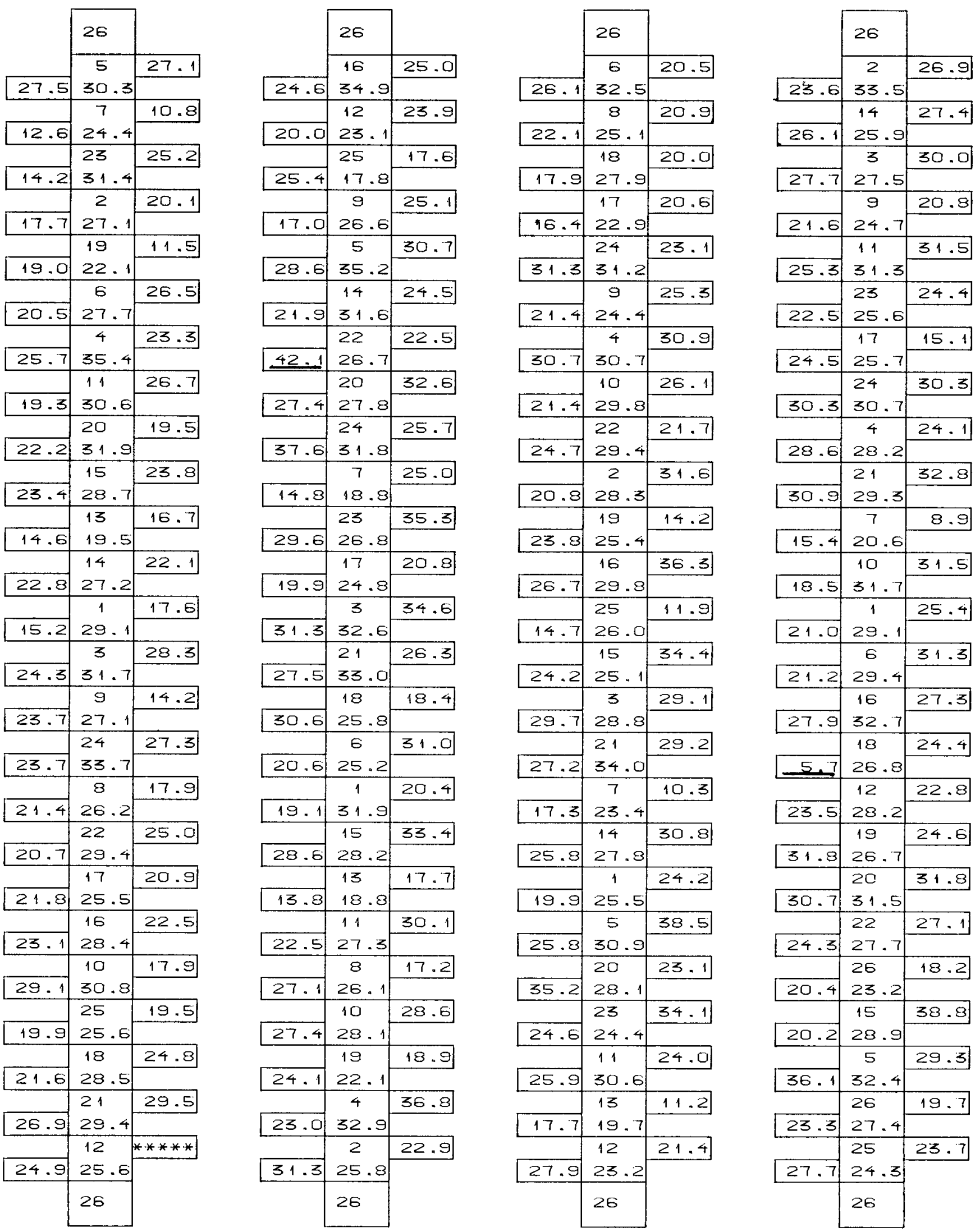

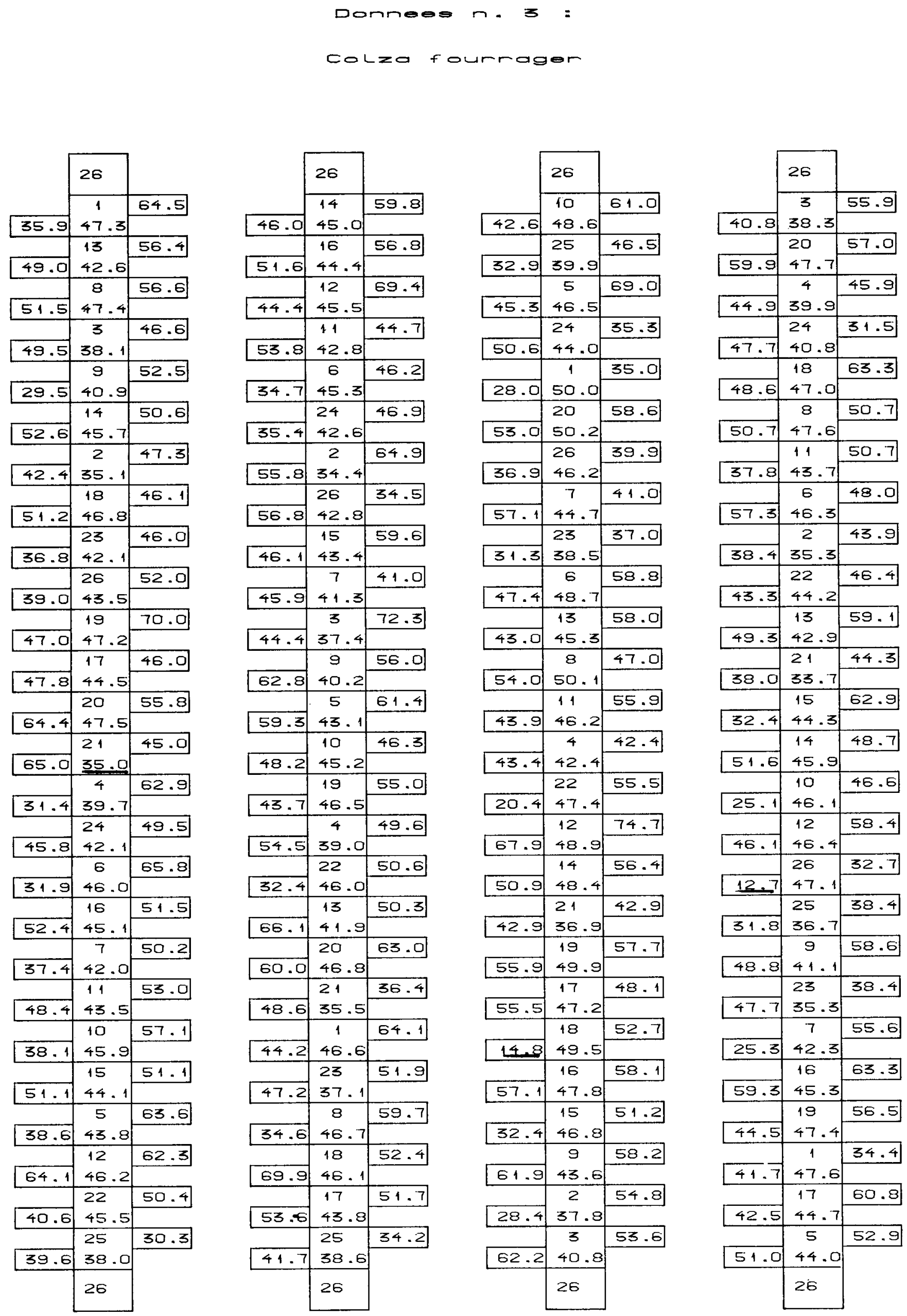
1 analyse de variance de ces données conduit aux estimations suivantes pour les effets producteur PR, compétiteur $\mathrm{C}$ et plein champ t.

TABLEAU 1

Essai 1 - Valeurs estimées des différents paramètres pour le colza oléagineux d'hiver. Trial 1 - Estimation of parameters for winter rapeseed.

\begin{tabular}{|c|c|c|c|c|c|c|c|}
\hline$n$ & PRi & $\mathrm{Ci}$ & $\mathrm{ti}$ & PR & $\begin{array}{l}\text { Moyennes } \\
\text { C }\end{array}$ & $\mathrm{t}$ & $\begin{array}{l}\text { Moyenne } \\
\text { générale }\end{array}$ \\
\hline \multicolumn{8}{|c|}{ Lignées pures } \\
\hline 1 & $-\quad 1,79$ & $-2,73$ & $-4,52$ & & & & \\
\hline 2 & - $\quad 7,24$ & 2,48 & $-4,75$ & & & & \\
\hline 3 & $-\quad 9,33$ & 3,03 & $-6,30$ & $-7,50$ & 2,71 & $-4,79$ & \\
\hline 4 & $-15,63$ & 7,05 & $-8,58$ & & & & \\
\hline 5 & $-\quad 0,19$ & $-0,12$ & $-0,32$ & & & & \\
\hline 6 & $-10,84$ & 6,57 & $-4,27$ & & & & \\
\hline & & & & & & & 21,6 \\
\hline \multicolumn{8}{|l|}{ Hybrides } \\
\hline 7 & $-0,71$ & $-2,41$ & $-3,12$ & & & & \\
\hline 8 & 2,00 & 2,05 & $-0,05$ & & & & \\
\hline 9 & 11,05 & $-2,75$ & 8,30 & & & & \\
\hline 10 & 5,93 & $-6,55$ & $-0,62$ & & & & \\
\hline 11 & 3,90 & $-1,26$ & 2,65 & 4,50 & $-1,63$ & 3,77 & \\
\hline 12 & 2,45 & $-2,27$ & 0,18 & & & & \\
\hline 13 & 5,28 & $-1,40$ & 3,88 & & & & \\
\hline 14 & 2,26 & 4,51 & 6,77 & & & & \\
\hline 15 & 7,10 & $-0,63$ & 6,47 & & & & \\
\hline 16 & 5,76 & $-1,48$ & 4,28 & & & & \\
\hline
\end{tabular}

TABLEAU 2

Essai 2 - Valeurs estimées des différents parainètres pour le colza oléagineux de printemps.

Trial 2 - Estimation of parameters for spring rapeseed.

\begin{tabular}{|c|c|c|c|c|c|c|c|}
\hline $\mathrm{n}$ & PRi & $\mathrm{Ci}$ & $\mathrm{ti}$ & PR & $\begin{array}{l}\text { Moyennes } \\
\text { C }\end{array}$ & $t$ & $\begin{array}{l}\text { Moyenne } \\
\text { générale }\end{array}$ \\
\hline \multicolumn{8}{|c|}{ Lignées pures } \\
\hline 1 & $-\quad 1,64$ & 2,23 & 0,59 & & & & \\
\hline 7 & $-8,80$ & 3,24 & $-5,56$ & & & & \\
\hline 13 & $-7,03$ & $-0,90$ & $-7,93$ & $-5,30$ & 1,76 & $-3,54$ & \\
\hline 19 & $-\quad 3,88$ & 1,11 & $-2,77$ & & & & \\
\hline 25 & $-\quad 5,59$ & 2,03 & $-3,56$ & & & & \\
\hline 26 & $-4,86$ & 2,83 & $-2,03$ & & & & \\
\hline \multirow{2}{*}{\multicolumn{8}{|c|}{ Hybrides }} \\
\hline & & & & & & & \\
\hline 2 & 1,49 & $-1,69$ & $-0,20$ & & & & \\
\hline 3 & 5,59 & $-2,85$ & 2,74 & & & & \\
\hline 4 & 3,88 & 0,47 & 4,35 & & & & \\
\hline 5 & 6,47 & $-2,50$ & 3,97 & & & & \\
\hline 6 & 0,07 & 0,61 & 0,68 & & & & \\
\hline 8 & $-2,34$ & 0,25 & $-2,09$ & & & & \\
\hline 9 & $-2,44$ & 0,86 & $-1,58$ & & & & \\
\hline 10 & 0,52 & 1,49 & 2,01 & & & & \\
\hline 11 & 3,54 & $-1,99$ & 1,55 & & & & \\
\hline 12 & $-2,25$ & $-0,28$ & $-2,53$ & 1,59 & $-0,53$ & 1,06 & \\
\hline 14 & 1,27 & $-0,80$ & 0,47 & & & & \\
\hline 15 & 3,41 & $-2,55$ & 0,86 & & & & \\
\hline 16 & 2,77 & 0,37 & 3,14 & & & & \\
\hline 17 & $-\quad 2,87$ & $-0,41$ & $-3,29$ & & & & \\
\hline 18 & $-\quad 1,32$ & 1,10 & $-0,22$ & & & & \\
\hline 20 & 3,44 & $-0,44$ & 3,00 & & & & \\
\hline 21 & 5,07 & $-1,62$ & 3,44 & & & & \\
\hline 22 & $-0,21$ & 0,91 & 0,70 & & & & \\
\hline 23 & 1,86 & $-2,06$ & $-0,20$ & & & & \\
\hline 24 & 3,86 & 0,60 & 4,46 & & & & \\
\hline
\end{tabular}


TABLEAU 3

Essai 3 - Valeurs estimées des différents paramèıres pour le colza fourrager.

Trial 3 - Estimation of parameters for fodder rape.

\begin{tabular}{|c|c|c|c|c|c|c|c|}
\hline $\mathrm{n}$ & PRi & $\mathrm{Ci}$ & $\mathrm{ti}$ & PR & $\begin{array}{l}\text { Moyennes } \\
\text { C }\end{array}$ & 1 & $\begin{array}{l}\text { Moyenne } \\
\text { générale }\end{array}$ \\
\hline \multicolumn{8}{|c|}{ Lignées pures } \\
\hline 21 & $-11,55$ & 3,22 & 8,33 & & & & \\
\hline 22 & $-1,72$ & 3,80 & 2,08 & & & & \\
\hline 23 & $-9,50$ & 4,11 & $-5,40$ & $-7,11$ & 4,25 & $-2,86$ & \\
\hline 24 & $-\quad 3,22$ & 1,88 & $-1,34$ & & & & \\
\hline 25 & $-12,04$ & 6,64 & $-5,40$ & & & & \\
\hline 26 & $-4,65$ & 5,86 & 1,21 & & & & \\
\hline & & & & & & & 47,26 \\
\hline \multicolumn{8}{|l|}{ Hybrides } \\
\hline 1 & $-\quad 4,18$ & 8,36 & 4,18 & & & & \\
\hline 2 & $-4,12$ & $-3,84$ & - 7,96 & & & & \\
\hline 3 & 1,31 & $-6,26$ & $-4,97$ & & & & \\
\hline 4 & $-\quad 2,62$ & $-0,80$ & $-3,42$ & & & & \\
\hline 5 & 3,82 & $-3,10$ & 0,73 & & & & \\
\hline 6 & 2,80 & 0,08 & 2,88 & & & & \\
\hline 7 & $-\quad 4,34$ & 3,26 & $-1,08$ & & & & \\
\hline 8 & 4,36 & $-0,12$ & 4,24 & & & & \\
\hline 9 & 2,60 & $-4,78$ & $-2,18$ & & & & \\
\hline 10 & $-\quad 1,28$ & 4,06 & 2,77 & & & & \\
\hline 11 & 1,98 & $-1,61$ & 0,37 & & & & \\
\hline 12 & 12,16 & $-9,07$ & 3,10 & 2,13 & $-1,27$ & 0,86 & \\
\hline 13 & 2,56 & $-3,04$ & $-0,48$ & & & & \\
\hline 14 & 4,77 & $-2,23$ & 2,54 & & & & \\
\hline 15 & $-\quad 0,74$ & 1,73 & 0,99 & & & & \\
\hline 16 & 6,89 & $-4,88$ & 2,01 & & & & \\
\hline 17 & 0,75 & 0,62 & 1,37 & & & & \\
\hline 18 & 4,85 & $-1,18$ & 3,67 & & & & \\
\hline 19 & 3,90 & 0,15 & 4,04 & & & & \\
\hline 20 & 7,23 & $-2,84$ & 4,39 & & & & \\
\hline
\end{tabular}

\section{RÉFÉRENCES BIBLIOGRAPHIQUES}

Astier et al., 1982. Genstat : un langage statistique. INRA, ouvrage collectif ISBN 2-85340-425-0, 555 p.

Azais J.-M., Denis J. B., Kobilinsky A., 1984. Première idè sur la compétition interparcellaire. Publication interne, Laboratoire de Biométrie, INRA, 78000 Versailles, $20 \mathrm{p}$.

Buson M., 1979. Hétérosis et paramètres génétiques de quelques caractères agronomiques chez le colza oléagineux (Brassica napus L.). Thèse de $3^{\mathrm{e}}$ cycle, Paris VI, $78 \mathrm{p}$.

Draper N., Smith H., 1966. Applied regression analysis. Wiley interscience, N. Y., 408 p.

Durbin J., Watson G. S., 1950. Testing for serial correlation in least square regression I. Biometrika, 37, 409-428.

Durbin J., Watson G. S., 1951. Testing for serial correlation in least square regression II. Biometrika, 38, 159-178.
Durbin J., Watson G. S., 1971. Testing for serial correlation in least square regression III. Biometrika, 58, 1-19.

Kobilinsky A., 1982. MODLI, MODULAD, INRIA Rocquencourt, 78150 Le Chesnay, p. 197-262.

Lefort-Buson M., 1982. Heterosis with spring rapeseed (Brassica napus L.). Cruciferae Newsl., 7, 16-17.

Lefort-Buson M., 1983. Preliminary results for fodder rape hybrids. Cruciferae Newsl., 8, 14-15.

Lefort-Buson M., Dattée Y., 1985. Etude de l'hétérosis chez le colza oléagineux d'hiver (Brassica napus L.). I. Comparaison de 2 populations, l'une homozygote et l'autre hétérozygote. Agronomie, 5 (2), 101-110. 